\title{
ESTUDO COM IDOSOS DE, INSTITUICCÕES ASILARES NO MUNICÍPIO DE NATAL/RN: CARACTERÍSTICAS SOCIOECONÔMICAS E DE SAÚDE
}

\author{
Rejane Marie Barbosa Davim ${ }^{1}$ \\ Gilson de Vasconcelos Torres ${ }^{2}$ \\ Susana Maria Miranda Dantas ${ }^{3}$ \\ Vilma Maria de Lima $^{4}$
}

Davim RMB, Torres GV, Dantas SMM, Lima VM. Estudo com idosos de instituições asilares no município de Natal/RN: características socioeconômicas e de saúde. Rev Latino-am Enfermagem 2004 maio-junho; 12(3):518-24.

O envelhecimento populacional acelerado no Brasil certamente aumentará o número de idosos institucionalizados. Baseados nesse enfoque, este estudo exploratório descritivo foi desenvolvido em três instituições asilares na cidade de Natal (RN) e objetivou caracterizar o idoso asilado; identificar os problemas socioeconômicos, de saúde e as causas que os levaram ao asilo. A amostra constituiu-se de $30 \%$ da totalidade de idosos de cada instituição, as quais têm caráter filantrópico e abrigam idosos carentes. Os resultados mostraram que as três instituições possuem características socioeconômicas e de saúde semelhantes às encontradas na literatura, pontuando-se baixas condições financeiras, contato familiar conflituoso, atividades de lazer limitadas ou ausentes, saúde precária, restrito atendimento médico e de enfermagem, além da ausência de planos privados de saúde. Conclui-se, refletindo-se quanto às condições socioeconômicas e de saúde desses idosos, que há a necessidade da atuação de órgãos competentes com vistas a seus direitos de cidadania.

DESCRITORES: asilo; idoso; saúde do idoso; características da população

\section{STUDY WITH ELDERLY FROM ASYLUMS IN NATAL/RN: SOCIOECONOMIC} AND HEALTH CHARACTERISTICS

The accelerated aging of the Brazilian population will certainly increase the number of institutionalized elderly. Based on this focus, this descriptive and exploratory study was carried out at three asylum institutions in Natal (RN), Brazil, with a view to characterizing the elderly living there, as well as identifying the socioeconomic and health problems and causes that took them to the asylum. The sample consisted of $30 \%$ of the total number of elderly in each asylum. These are philanthropic institutions and give shelter to poor elderly persons. Results showed that the three institutions' socioeconomic and health characteristics were similar to what is found in literature, with few financial conditions, family contact marked by conflict, lack or absence of leisure activities, precarious health, restricted medical and nursing care and absence of private health plans. Our reflections on the socioeconomic and health conditions of these persons led to the conclusion that public bodies need to take actions to safeguard their civil rights.

DESCRIPTORS: asylum; aged; health of the elderly; population characteristics

\section{ESTUDIO CON ANCIANOS DE INSTITUCIONES ASILARES EN EL MUNICIPIO DE NATAL/RN: CARACTERÍSTICAS SOCIO-ECONÓMICAS Y DE SALUD}

El envejecimiento poblacional acelerado en Brasil ciertamente aumentará el número de ancianos institucionalizados. Basado en este hecho, este estudio exploratorio descriptivo fue realizado en tres instituciones asilares en Natal (RN), Brasil, con la finalidad de caracterizar al anciano que vive en un asilo, identificándose las características socioeconómicas y los problemas de salud afrontados por ellos y las causas de su institucionalización. La muestra consiste en 30\% de la cantidad total de ancianos de cada institución. Los asilos son instituciones filantrópicos y albergan al anciano necesitado. Los resultados mostraron que las tres instituciones tenían características socioeconómicas y de salud similares a lo que se encuentra en la literatura, tales como las bajas condiciones financieras, contacto familiar conflictivo, actividades de ocio limitadas o ausentes, condición de salud precaria, ayuda médica y de enfermería restringida y ausencia de planes de salud privados. Nuestros reflexiones acerca de las condiciones socioeconómicas y de salud llevaron a la conclusión que son necesarios acciones de los órganos competentes para garantir los derechos civiles.

DESCRIPTORES: asilo; anciano; salud del anciano; características de la población

\footnotetext{
${ }^{1}$ Enfermeira Obstetra, Mestre em Enfermagem de Saúde Pública pela Universidade Federal da Paraíba, Doutoranda em Ciências da Saúde, Professor Adjunto, e-mail: rejanemb@uol.com.br; ${ }^{2}$ Enfermeiro, Doutor em Enfermagem pela Escola de Enfermagem de Ribeirão Preto, da Universidade de São Paulo, Professor Adjunto; ${ }^{3}$ Enfermeira, Mestre em Enfermagem Médico Cirúrgica pela Universidade do Estado do Rio de Janeiro, Professor Adjunto; ${ }^{4}$ Enfermeira, Mestre em Ciências da Enfermagem pela Universidade do Rio de Janeiro, Professor Adjunto. Universidade Federal do Rio Grande do Norte
} 
INTRODUÇÃO

Possuindo características próprias em sua estrutura social, a velhice coloca-nos como sujeitos e agentes da saúde para abrir espaços e vivenciar novas experiências, levando-se em consideração que o envelhecimento possui múltiplas dimensões, as quais abrangem questões de ordem social, política, cultural e econômica. Assim, essas questões relativas ao envelhecimento humano têm sido tema de relevante importância, uma vez que, nos países em desenvolvimento, como o Brasil, a estimativa de vida das pessoas tem aumentado de forma significativa.

O envelhecimento da população é um fenômeno de amplitude mundial, a OMS (Organização Mundial de Saúde) prevê que, em 2025, existirão 1,2 bilhões de pessoas com mais de 60 anos, sendo que os muitos idosos (com 80 ou mais anos) constituem o grupo etário de maior crescimento. No Brasil, estima-se que haverá cerca de 34 milhões de idosos em 2025, o que levará o Brasil à $6^{a}$ posição entre os países mais envelhecidos do mundo ${ }^{(1-2)}$

Essas projeções são baseadas em estimativas conservadoras quanto à fecundidade e mortalidade, sendo que, se ocorrer uma acentuada melhoria nas condições sociais, nas zonas mais carentes, como o Nordeste, o envelhecimento da população brasileira ocorrerá em maiores proporções ${ }^{(2)}$. Isso pode afetar diretamente, e de forma significativa, a estrutura etária da população e, conseqüentemente, vão aumentar, em grande parte, os problemas de uma determinada sociedade ${ }^{(3)}$.

$\mathrm{Na}$ realidade, existe uma grande preocupação com o idoso, nas diversas áreas, pois ele constitui um grupo bastante diferenciado entre si e em relação aos demais grupos etários, e os custos econômicos e sociais decorrentes deles vêm transcendendo as projeções para esse grupo, numa situação vigente do sistema espoliado de saúde pública.

Dessa forma, o crescimento da população idosa no país deve merecer, cada vez mais, o interesse dos órgãos públicos, das políticas sociais e da sociedade em geral, levando-se em consideração, principalmente, as características demográficas, econômicas, sociais e de saúde do país.

À medida que a idade avança, existe uma progressiva perda de recursos físicos, mentais e sociais, a qual tende a despertar sentimentos de desamparo. A velhice parece deixar o indivíduo impotente, indefeso, fragilizado para tomar suas próprias decisões, para enfrentar seus problemas, o cotidiano, não só diante dos familiares, mas também da sociedade como um todo. Sendo assim, o idoso tem sido visto como uma pessoa improdutiva, ultrapassada, e pouco se tem feito para recuperar sua identidade e elevar sua auto-estima. Além disso, nem sempre é amparado pelos familiares e, muitas vezes, são obrigados a morar em asilos ou albergues, forçados a viverem isolados, na solidão, longe de parentes e amigos.

A situação familiar do idoso no Brasil reflete o efeito cumulativo em eventos socioeconômicos, demográficos e de saúde ao longo dos anos, demonstrando que o tamanho da prole, as separações, 0 celibato, a mortalidade, a viuvez, os recasamentos e as migrações, vão originando, no desenvolver das décadas, tipos de arranjos familiares e domésticos, onde o morar sozinho, com parentes ou em asilos, pode ser o resultado desses desenlaces.

Os asilos, geralmente, são casas inapropriadas e inadequadas às necessidades do idoso, as quais não Ihes oferecem assistência social, cuidados básicos de higiene e alimentação. Ademais, esses locais vêm também dificultar as relações interpessoais no contexto comunitário, indispensáveis à manutenção do idoso pela vida e pela construção de sua cidadania ${ }^{(4)}$. Constituem, também, a modalidade mais antiga e universal de atendimento ao idoso, fora do seu convívio familiar, tendo como, inconveniente, favorecer seu isolamento, sua inatividade física e mental, tendo, dessa forma, conseqüências negativas à sua qualidade de vida ${ }^{(5)}$.

No entanto, o Decreto ํo 1.948 de 03 de julho de $1996^{(6)}$, frisa, no artigo $3^{\circ}$, que a instituição asilar tem, por finalidade, atender, em regime de internato, o idoso sem vínculo familiar ou sem condições de prover a própria subsistência, de modo a satisfazer suas necessidades de moradia, alimentação, saúde e convivência social( ${ }^{(7)}$. Prioriza, também, a Lei 8.842 , de janeiro de $1994^{(8)}$, no artigo $4^{\circ}$, parágrafo III, atendimento ao idoso pelas famílias, ao invés do asilar. Porém, com a existência de vários fatores, tais como os demográficos, sociais e de saúde, conduzem ao aumento da demanda pela institucionalização.

No Brasil, o suporte provido pela família - base principal do apoio oferecido ao idoso pelo tripé famíliacomunidade-Estado - como na maioria dos países em 
desenvolvimento, deverá enfrentar dificuldades crescentes como: 1) a não existência de políticas sociais de suporte aos cuidadores, em referência aos familiares ou outros indivíduos que prestam auxílio direto ao idoso em suas atividades básicas, a saber: alimentação, auxílio domiciliar, assistência médica e serviços de orientação, entre outros; 2) o tamanho das famílias no Brasil, que vem diminuindo devido à queda da fecundidade; 3) o aumento na proporção de separações conjugais, idosos residindo sozinhos, casais que optam por não ter filhos, e mães que criam sozinhas seus filhos; 4) idosos residindo com familiares cuja renda total não ultrapassa três salários mínimos e 5) por fim, o sistema de suporte formal que não tem sido capaz de substituir o papel da família ${ }^{(9)}$.

A problemática do idoso também está relacionada às aposentadorias precoces, com baixo poder aquisitivo, as quais constituem sua principal fonte de rendimentos, impossibilitando o atendimento de suas necessidades; discriminação nos serviços de saúde; exclusão do idoso na família e comunidade, devido à indefinição de uma política de valorização dessa população ${ }^{(10)}$.

Diante dessa situação e à medida que a população envelhece, aumenta a demanda por instituições de longa permanência. Nos Estados Unidos (EUA), cerca de 5\% dos idosos residem em abrigos que oferecem serviços de saúde, lazer e assistência social. Na Inglaterra, a freqüência de institucionalizações é minimizada por meio do atendimento em hospitais-dia, com assistência multidisciplinar à saúde, oferecida a essa população, principalmente na área da reabilitação, e elas prestamse, em grande parte, para "aliviar" o trabalho extra dos familiares de idosos dependentes ${ }^{(9)}$.

Na maioria das vezes, os asilos costumam surgir, espontaneamente, das necessidades sociais da comunidade, ocorrendo, nesse caso, problemas na qualidade de vida que os residentes lá encontram. Dessa forma, os principais serviços existentes oferecidos a essa população dirigem-se à saúde, sendo comum, em grande parte das capitais do país, instituições asilares privadas ou filantrópicas direcionadas ao idoso, e, com raras exceções, aquelas mantidas pelo Estado.

No Brasil, embora grande proporção de idosos institucionalizados seja dependente por problemas físicos ou mentais, a miséria e o abandono são os principais motivos da institucionalização, e a maioria, em especial nas regiões metropolitanas de Belo Horizonte e São Paulo, é mantida por instituições de caridade como a Sociedade
São Vicente de Paulo (SSVP) ${ }^{(9)}$.

Quanto às características das instituições asilares dirigidas ao idoso, normalmente são locais com espaço e áreas físicas semelhantes a grandes alojamentos. Raras são as que mantêm pessoal especializado para assistência social e à saúde ou que possuam uma proposta de trabalho voltada para manter o idoso independente e autônomo. Eles vivem, na maioria das vezes, como se estivessem em reformatórios ou internatos, com regras de entradas e saídas, poucas possibilidades de vida social, afetiva e sexual ativa. Na realidade, muitas vezes o que se encontra são depósitos de pessoas, que, fundamentados na idéia de amor ao próximo e amparo aos desabrigados, consideram que os abrigos, juntamente com os cuidados a eles prestados, são suficientes às pessoas que estejam em seus últimos dias de vida ${ }^{(11)}$.

Com vistas a toda essa problemática, no que se refere ao idoso, o crescimento dessa população tem sido objeto de estudo para alguns autores ${ }^{(12-13)}$, os quais afirmam que $o$ asilo não deveria ser configurado apenas como uma instituição que acolhe idosos rejeitados ou abandonados pela família, mas que também deve ser lembrada, compreendida e respeitada como uma escolha dentro de um contexto de vida de cada indivíduo.

Na mesma direção, o direito à cidadania para o idoso ainda é um sonho, pois a sua maioria vive como cidadão de segunda classe, sem condição de exercer sua cidadania. Assim, é drástica a situação do indivíduo asilado, afastado do convívio social, à mercê de uma vida padronizada, desprovida de prazer e de importância pessoal, levando-o à falta de perspectiva e à alienação ${ }^{(14)}$.

Diante do exposto, este estudo teve, como objetivos, caracterizar a realidade dos idosos de três instituições asilares, no município de Natal/RN, identificar os problemas sociais e de saúde enfrentados por eles e as causas que os levaram à institucionalização.

Ademais, o estudo justifica-se à medida que proporciona uma reflexão sobre as questões socioeconômicas e de saúde de idosos asilados, buscando despertar o interesse e a atenção dos cuidadores dessa população quanto à inserção deles no contexto social, econômico e de saúde.

Denota-se, ainda, significativa relevância científica e social no campo da enfermagem e da equipe de saúde, uma vez que representa a possibilidade de contribuição ao trabalho com idosos, o avanço no campo da pesquisa, enfocando, da mesma forma, a importância das relações 
interpessoais no contexto asilar, indispensável à qualidade de vida e à cidadania do indivíduo idoso, no intuito de promover o bem-estar das pessoas maduras, particularmente, no nosso contexto.

\section{MÉTODO}

Trata-se de um estudo descritivo e exploratório, com uma abordagem quantitativa, realizado em instituições asilares para idosos, na cidade de Natal/RN, buscando-se, na revisão de literatura, compreender o contexto social, econômico e de saúde a que esses indivíduos estão inseridos.

Inicialmente, realizou-se levantamento, junto à Secretaria Municipal de Saúde de Natal, com a finalidade de identificar as instituições asilares cadastradas pelo Departamento de Apoio à Pessoa Idosa da Secretaria Municipal de Promoção Social, encontrando-se três instituições.

Essas instituições foram caracterizadas como $A$, $\mathrm{B}$, e $\mathrm{C}$, com a finalidade de manter o seu sigilo e de seus residentes, ou seja, os idosos, e têm como função a assistência filantrópica, objetivando abrigo ao idoso carente, buscar soluções para seus problemas, desenvolver-lhes a saúde preventiva e curativa. A instituição A conta com 170 idosos, sendo 72 homens e 98 mulheres; a B, com 47, desses, 19 são masculinos, e 28, femininos; por fim, a C, que funciona com 42 idosas. Os recursos financeiros dessas instituições são adquiridos por meio de doações das Secretarias Estadual e Municipal, da população e dos próprios idosos, com suas aposentadorias.

Fez-se contato com os diretores das instituições, com o objetivo de permitirem a realização do estudo. A autorização foi formulada, e a coleta de informações teve seu início após consentimento livre e esclarecido dos participantes, segundo a Resolução 196/96, do Conselho Nacional de Saúde, que dispõe sobre pesquisa realizada com seres humanos.

A população foi constituída por uma amostra escolhida de forma aleatória estratificada, por meio de um percentual de $30 \%$ da totalidade de idosos de cada instituição, representando assim, 76 participantes para a coleta, utilizando-se, para isso, um formulário estruturado, e tendo-se, como técnica, a entrevista. Ele constou de quatro partes: a primeira, relacionada à identificação do idoso; a segunda, explorando seus aspectos sociais; a terceira, identificando os problemas de saúde, e a última, questionando aspectos quanto à permanência do idoso na instituição.

As entrevistas foram realizadas nos próprios asilos, observando-se os aspectos éticos e legais no que se refere ao anonimato dos entrevistados. A análise dos dados foi baseada na estatística descritiva à luz das informações colhidas, dos objetivos propostos e da revisão de literatura.

\section{RESULTADOS E DISCUSSÃO}

Caracterizando a população

Priorizam-se alguns aspectos relevantes para caracterizar os idosos das instituições pesquisadas. Nesse sentido, $58 \%$ dos pesquisados, nas instituições $A$ e $B$, são do sexo feminino e, na $C$, representam sua totalidade.

Tais resultados confirmam a tendência nos estudos relacionados com os idosos, pois a participação masculina raramente ultrapassa os $20 \%$, parecendo ser, no Brasil, uma experiência essencialmente feminina. Ressalta-se, ainda, que, no Brasil, o número absoluto de mulheres idosas tem sido superior quando confrontado com o de homens de 65 anos ou mais. Isso pode acontecer pela existência da mortalidade diferencial de sexo, que prevalece, há longas datas, na população brasileira ${ }^{(15)}$

Quanto à faixa etária, 30\% da instituição $\mathrm{A}$ e $\mathrm{B}$ têm mais de 80 anos, enquanto, na C, 50\% têm de 60 a 64 anos. Estudos relatam que a faixa etária representa ponto importante na população idosa, pelo maior risco em adoecer e apresentar maior grau de dependência, quanto mais avançada ela se apresenta ${ }^{(16)}$. Quanto ao estado civil, $48 \%$ são solteiros, em A e C, e $79 \%$, viúvos ou separados, em B. Nas instituições A e C, $97 \%$ são católicos, e $71 \%$ da $B$ são evangélicos.

Foi baixo o nível de escolaridade, pois $53 \%$ dos idosos das instituições $A$ e $B$ têm o $1^{\circ}$ grau, enquanto $46 \%$ do total de idosos pesquisados não são alfabetizados. Esses percentuais mostram o grande número de pessoas com pouco ou nenhum grau de escolaridade, pois o analfabetismo no idoso representa uma realidade nos países em desenvolvimento, como, por exemplo, o Brasil, principalmente quando se trata de idosos que viveram sua 
infância em época em que o ensino não era prioridade, principalmente com relação à mulher ${ }^{(17)}$.

O grau de escolaridade implica que a assistência de enfermagem considere quando as atividades educativas são realizadas, concomitante a outras alterações específicas do envelhecimento, como, por exemplo, a diminuição da acuidade visual e auditiva, por interferirem na aprendizagem dessa população. É importante, também, ressaltar que a equipe de enfermagem deve estar atenta a essa baixa escolaridade, para que a linguagem usada com esses idosos seja a mais acessível possível, para que eles consigam entender o significado da informação passada e realizem a prática do cuidado com sucesso.

Relacionando-se os aspectos sociais, $87 \%$ trabalhavam anteriormente, $97 \%$ afirmaram receber benefícios por meio de doações. Do total de idosos, $91 \%$ recebiam aposentadoria de 1 salário mínimo, e 20\% dos residentes da instituição $A$ referiram receber auxílio financeiro de familiares. Diante dessa situação, os idosos pesquisados não conseguem seguir adequadamente as orientações médicas com relação aos medicamentos, e, principalmente, com relação à dieta alimentar. Outros fatores também vão interferir diretamente no cuidar, como vestuário, higiene, entre outros ${ }^{(18)}$

No aspecto familiar, $83 \%$ têm familiares; destes, $45 \%$ residem em Natal, e 38\%, no interior do RN. Quanto às visitas, $67 \%$ afirmaram que são visitados por filhos (37\%), sobrinhos e irmãos (24\%). Dentre os pesquisados, $62 \%$ informaram que não gostariam de estar junto aos familiares (B e C), porém 58\% da instituição A mostraram o desejo desse convívio.

No que se refere ao lazer, $69 \%$ dos idosos das instituições $\mathrm{A}$ e $\mathrm{C}$ participam de atividades de lazer, porém $100 \%$ da B são totalmente sedentários. Questionados quanto à importância do lazer, $67 \%$ dos idosos consideram essa atividade necessária em suas vidas, enquanto $49 \%$ informaram que não gostariam de fazer nada.

Salienta-se que, mesmo estando aposentado, o ser humano não deve acomodar-se, devido ao sedentarismo. É importante que esse indivíduo desempenhe atividades de acordo com sua vontade e habilidade pessoal, visando a uma contribuição para o prolongamento da vida, tornando-a mais prazerosa, pela realização de um projeto de vida.

Nos aspectos relacionados à saúde, pode-se observar que $66 \%$ dos pesquisados na instituição C consideram seu estado de saúde de muito bom a bom; $86 \%$ na $\mathrm{B}$ e $74 \%$ na $A$ responderam de mais ou menos a péssimo. Os problemas mais citados pelos idosos foram dor nas articulações (63\%), problemas de vista (59\%) e doenças do coração (31\%).

\section{Aspectos relacionados à saúde}

$\mathrm{Na}$ Tabela a seguir, mostram-se os aspectos relacionados à saúde, citados pelos idosos pesquisados nas três instituições asilares.

Tabela 1 - Distribuição dos aspectos relacionados à saúde dos idosos pesquisados em três instituições asilares. Natal/RN - 1999

\begin{tabular}{|c|c|c|c|c|c|c|}
\hline \multirow{3}{*}{$\begin{array}{l}\text { Aspectos relacionados à } \\
\text { saúde }\end{array}$} & \multicolumn{6}{|c|}{ INSTITUIÇŌES } \\
\hline & \multicolumn{2}{|c|}{$A=50$} & \multicolumn{2}{|c|}{$B=14$} & \multicolumn{2}{|c|}{$C=12$} \\
\hline & $\mathrm{N}$ & $\%$ & $\mathrm{~N}$ & $\%$ & $\mathrm{~N}$ & $\%$ \\
\hline \multicolumn{7}{|l|}{ 1-Alimenta-se sozinho? } \\
\hline Sim & 50 & 100 & 13 & 93 & 12 & 100 \\
\hline Com ajuda & --- & -- & 01 & 07 & --- & -- \\
\hline \multicolumn{7}{|l|}{ 2- Caminha c/ facilidade? } \\
\hline Sim & 29 & 58 & 07 & 50 & 11 & 92 \\
\hline Não & 11 & 22 & 02 & 14 & 01 & 08 \\
\hline Com ajuda (outras & 10 & 20 & 05 & 36 & --- & --- \\
\hline \multicolumn{7}{|l|}{$\begin{array}{l}\text { pessoas, bengala, cadeira de } \\
\text { rodas) } \\
3 \text {-Dorme bem? }\end{array}$} \\
\hline Sim & 34 & 68 & 11 & 79 & 10 & 83 \\
\hline Não & 16 & 32 & 03 & 21 & 02 & 17 \\
\hline \multicolumn{7}{|l|}{ 4- Possui hábito de fumar? } \\
\hline Sim (cigarro, cachimbo) & 11 & 22 & --- & --- & --- & --- \\
\hline Não & 39 & 78 & 14 & 100 & 12 & 100 \\
\hline 5 - Possui hábito de beber? & & & & & & \\
\hline Não & 50 & 100 & 14 & 100 & 12 & 100 \\
\hline
\end{tabular}

Conforme os dados acima, apenas 01 idoso da instituição B referiu necessitar de ajuda para alimentarse. Observa-se, da mesma forma, que $92 \%$ dos idosos da instituição $C$ afirmaram caminhar com facilidade, no entanto $36 \%$ da B e $20 \%$ da A necessitam de ajuda para se locomoverem. Quanto ao sono, a maioria dos idosos dorme bem; em relação ao fumo, apenas $22 \%$ da instituição A referiram esse hábito, e 100\% dos residentes nos três asilos não ingerem bebidas alcoólicas.

No que se refere à assistência médica, $76 \%$ dos pesquisados da instituição A e $100 \%$ da C afirmaram procurar assistência médica quando adoecem, enquanto os da instituição B referiram o contrário, ou seja, não procuram o médico. Ao serem interrogados sobre quem cuida deles quando adoecem, $100 \%$ dos idosos da instituição $C$ procuram o médico e enfermeiro, $93 \%$ da $B$ recebem os cuidados de auxiliares de enfermagem, e 70\% da A têm esses cuidados do profissional médico. $100 \%$ 
dos idosos das instituições B e C e 96\% da A afirmaram não possuírem plano de saúde privado.

\section{Aspectos sociais}

Tabela 2 - Distribuição dos aspectos sociais relacionados às instituições dos idosos pesquisados. Natal/RN - 1999

\begin{tabular}{|c|c|c|c|c|c|c|}
\hline \multirow{3}{*}{$\begin{array}{c}\text { Aspectos sociais relacionados } \\
\text { às instituições }\end{array}$} & \multicolumn{6}{|c|}{ INSTITUIÇŌES } \\
\hline & \multicolumn{2}{|c|}{$A=50$} & \multicolumn{2}{|c|}{$B=14$} & \multicolumn{2}{|c|}{$C=12$} \\
\hline & $\mathrm{N}$ & $\%$ & $\mathrm{~N}$ & $\%$ & $\mathrm{~N}$ & $\%$ \\
\hline \multicolumn{7}{|l|}{ 1. Sente-se bem na instituição } \\
\hline Sim & 39 & 78 & 10 & 71 & 11 & 92 \\
\hline Não (não gosta do local; as & 11 & 22 & 04 & 29 & 01 & 08 \\
\hline $\begin{array}{l}\text { pessoas são más; muito } \\
\text { barulho; não gosta dos colegas) }\end{array}$ & & & & & & \\
\hline \multicolumn{7}{|l|}{ 2. Por que veio morar aqui? } \\
\hline Problemas de família & 18 & 36 & 09 & 64 & 06 & 50 \\
\hline Não tinha para onde ir & 12 & 24 & 02 & 14 & 02 & 16 \\
\hline Motivo de doença & 06 & 12 & 03 & 21 & 03 & 25 \\
\hline Morava só & 10 & 20 & -- & -- & -- & -- \\
\hline Problemas conjugais & 03 & 06 & -- & -- & 01 & 08 \\
\hline Estava perdido na rua & 01 & 02 & -- & -- & -- & -- \\
\hline \multicolumn{7}{|l|}{ 3. Quem o trouxe para cá? } \\
\hline Veio só & 15 & 30 & 04 & 28 & 01 & 08 \\
\hline O irmão & 07 & 14 & 03 & 21 & 04 & 33 \\
\hline O filho & 08 & 16 & 02 & 14 & -- & -- \\
\hline Um conhecido & 07 & 14 & 02 & 14 & 02 & 17 \\
\hline Parentes & 06 & 12 & 02 & 14 & 03 & 25 \\
\hline Os patrões & 04 & 08 & -- & -- & 02 & 17 \\
\hline A assistente social & 02 & 04 & 01 & 07 & -- & -- \\
\hline A polícia & 01 & 02 & -- & -- & -- & -- \\
\hline \multicolumn{7}{|l|}{$\begin{array}{l}\text { 4. Com quem morava antes de } \\
\text { vir para a instituição? }\end{array}$} \\
\hline Com a família & 24 & 48 & 07 & 50 & 07 & 50 \\
\hline Sozinho & 11 & 22 & 07 & 50 & 07 & 50 \\
\hline Com marido/esposa & 08 & 16 & -- & -- & -- & -- \\
\hline Patrões & 04 & 08 & -- & -- & -- & -- \\
\hline Amigos & 03 & 06 & -- & -- & -- & -- \\
\hline \multicolumn{7}{|l|}{$\begin{array}{l}\text { 5. Gostaria de morar em outro } \\
\text { lugar? }\end{array}$} \\
\hline $\begin{array}{c}\text { Sim (minha casa; interior; } \\
\text { qualquer lugar, praia) }\end{array}$ & 24 & 48 & 06 & 43 & 04 & 33 \\
\hline $\begin{array}{l}\text { Não (gosta da instituição; } \\
\text { não tem para onde ir; } \\
\text { está velho e ninguém } \\
\text { quer) }\end{array}$ & 26 & 52 & 08 & 57 & 08 & 67 \\
\hline
\end{tabular}

Conforme a Tabela 2, observa-se que a maioria dos idosos pesquisados afirmaram sentir-se bem nas instituições onde residem. Quando questionados sobre qual o motivo que os levaram às instituições, estas foram as respostas: problemas familiares, não tinham para onde ir, motivo de doença, entre outros.

Ao questionar-se com quem moravam antes de virem para as instituições, identifica-se que a maioria (48\%) dos idosos da instituição A residiam com familiares, e $22 \%$, sozinhos, enquanto nas instituições B e C, $50 \%$ residiam com familiares, e 50\%, sozinhos. Observa-se, também, que a maioria dos residentes das três instituições não gostariam de sair do convívio asilar, justificando gostar de morar no local, não tinham para onde ir ou mesmo por serem muito velhos e ninguém vai querer estar com eles.
O relacionamento desses idosos em instituição asilar torna-se um contexto "familiar", e eles encontram proteção tanto dos profissionais como dos colegas participantes. Dessa forma, ocorre uma substituição de elementos, uma vez que a convivência cotidiana familiar de origem torna-se, na maioria das vezes, esporádica (pais, irmãos), como também com a família construída (filhos). Assim, o cotidiano com os semelhantes que possuem experiências da mesma época, de partilhar essas experiências e os vínculos afetivos, mescla-os com os novos amigos, tornando-se a imagem tão forte que os idosos verbalizam a expressão "pegar amizade", tornandose, assim, uma manifestação de carinho e confiança encontrados por meio da participação no convívio asilar ${ }^{(19)}$.

\section{CONSIDERAÇÕES FINAIS}

Com o índice crescente da população idosa, as instituições de caráter asilar constituem-se, na maioria das vezes, opção ímpar para uma melhor qualidade de vida desses indivíduos. Porém, é necessário que eles recebam toda ajuda estrutural, tanto de recursos materiais quanto humanos, para se atender, da melhor forma possível, a essa população com características especiais.

Nas instituições asilares pesquisadas, predomina o caráter filantrópico assistencial, contando com idosos de ambos os sexos, recebendo recursos financeiros por meio de doações das Secretarias de Saúde do Estado e do Município de Natal/RN, como também das aposentadorias dos idosos residentes.

É importante destacar que os grupos pesquisados nas três instituições possuem características socioeconômicas e de saúde semelhantes às encontradas em outros estudos ${ }^{(20-21)}$, onde as mais marcantes são relacionadas ao baixo poder aquisitivo, contato familiar conflituoso, atividades de lazer limitadas e/ou ausentes nas instituições, como também condições de saúde precárias, restrito atendimento médico e de enfermagem, com a ausência de planos privados de saúde.

Os resultados apontam, também, que os problemas de saúde mais citados pelos idosos são referentes à dor nas articulações, dificuldades visuais e doenças do coração, implicando, dessa forma, a necessidade quanto à ajuda para alimentarem-se e caminharem sozinhos.

Argumentam, ainda, que, dentre as principais 
causas que os levaram a instalarem-se nas instituições, destacam-se: problemas familiares e de saúde, não tinham onde morar, entre outros. Vale ressaltar que a maioria desses idosos foi levada às instituições por familiares ou por iniciativa própria.

Outrossim, pontua-se a necessidade de uma atenção urgente à população em estudo, pois sabe-se que a tendência nos próximos anos é de seu aumento expressivo, em especial das instituições asilares, o que nos remete a repensar, discutir, refletir e encontrar formas

\section{REFERÊNCIAS BIBLIOGRÁFICAS}

1. Sousa L, Galante H, Figueiredo D. Qualidade de vida e bem-estar dos idosos: um estudo exploratório na população portuguesa. Rev Saúde Pública 2003 junho; 37(3):364-71. 2. Instituto Brasileiro de Geografia e Estatística (IBGE). Censo Demográfico 2000. Rio de Janeiro (RJ): Instituto Brasileiro de Geografia e Estatística; 2000.

3. Freitas MC, Maruyama SAT, Ferreira TF, Motta AMA. Perspectivas das pesquisas em gerontologia e geriatria: revisão da literatura. Rev Latino-am Enfermagem 2002 marçoabril; 10(2):221-8.

4. Vieira EB. Manual de gerontologia: um guia teórico-prático para profissionais cuidadores e familiares. Rio de Janeiro (RJ): Revinter; 1996.

5. Brito FC, Ramos LR. Serviços de atenção à saúde do idoso. In: Papaléo M Netto. Gerontologia. São Paulo (SP): Atheneu; 1996.

6. Decreto no 1.948 Regulamenta a Lei no 8.842 de 04 de janeiro de 1994 - Política Nacional do Idoso e dá outras providências. Diário Oficial da República Federativa do Brasil, 1996. 05 jan, seção 1, p. 77-9.

7. Louzã MR Netto, Louzã SPR, Cohen C, Louzã JR. O idoso, as instituições totais e a institucionalização. Rev Paul Hosp 1986 julho-agosto; 34(7/8//9):135-43.

8. Ministério da Previdência e Assistência Social (BR). Lei no 8.842 de 04 de janeiro de 1994. Dispõe sobre a política nacional do idoso e dá suas providências. Brasília (DF): Ministério da Previdência e Assistência Social; 1996.

9. Chaimowicz F. Os idosos brasileiros do século XXI: demografia, saúde e sociedade. Belo Horizonte (MG): Postgraduate; 1998.

10. Leme LEG. O envelhecimento. São Paulo (SP): Contexto; 1998.

11. Silva YA. A enfermagem nos serviços e programas públicos de atenção ao idoso. Texto \& Contexto Enfermagem 1997 maio-agosto; 6(2):127-36.

12. Duarte MJRS. Atenção ao idoso: um problema de saúde pública e de enfermagem. Rev Enfermagem UERJ 1994; 2(1):100-11.

13. Rodrigues RAP, Diogo MJD’E organizadores. Como cuidar dos idosos. Campinas (SP): Papirus; 1996.

14. Barros PMR. Oliveira MVC, Vasconcelos EMR. Política de atenção institucionalizada ao idoso. In: 50ํㅡㄹ Congresso Brasileiro de Enfermagem; 1998. setembro 20-25; Salvador, Bahia. Salvador: ABEn; 1998. p. 128. alternativas de integração social, convívio familiar e, sobretudo, o envolvimento efetivo dos órgãos públicos, da saúde e da sociedade como um todo, no enfrentamento dessa realidade.

Nessa perspectiva, considera-se este estudo como um despertar para as questões ligadas às instituições asilares dos idosos como ponto de reflexão, para entenderse melhor a problemática vivenciada por essa população, ou o seu processo de inserção nessas instituições.

15. Berquó E. Algumas considerações demográficas sobre o envelhecimento da população no Brasil. In: Anais do 1ํㅜ Seminário Internacional Envelhecimento Populacional: uma agenda para o final do século. Brasília (DF): MPAS/SAS; 1996. p. 16-34.

16. Marin MJS, Angerami ELS. Caracterização de um grupo de idosas hospitalizadas e seus cuidadores visando o cuidado pós-alta hospitalar. Rev Esc Enfermagem USP 2002 março; 36(1):33-41.

17. Kinsella K. Dimensiones demográficas y de salud en América Latina y el Caribe. In: Anzola PE, Galinski D, Morales MF, Salas AR, Sanches AM. La atención de los ancianos: un desafío para los años noventa. Washington: OPAS/OMS; 1994. p. 3-18.

18. Oliveira DR, Tuoto FS, Lenardt MH. As práticas de cuidado de si dos doentes idosos submetidos ao tratamento dialítico.Cogitare Enfermagem 2000 julho-dezembro; 5(2):7580.

19. Borini MLO, Cintra FA. Representações sociais da participação em atividades de lazer em grupos da Terceira Idade. Rev Bras Enferm 2002 setembro-outubro; 55(5):56874.

20. Yamamoto A, Diogo MJD’E. Os idosos e as instituições asilares do município de Campinas. Rev Latino-am Enfermagem 2002 setembro-outubro; 10(5):660-6.

21. Santos SR, Santos IBC, Fernandes MGM, Henriques MERM. Qualidade de vida do idoso na comunidade: aplicação da Escala de Flanagan. Rev Latino-am Enfermagem 2002 novembro-dezembro; 10(6):757-64. 